\title{
Minimum Principle-Type Necessary Optimality Conditions in Scalar and Vector Optimization. An Account
}

\author{
Giorgio Giorgi ${ }^{1}$ \\ ${ }^{1}$ Department of Economics and Management, Via S. Felice, 5 - 27100 Pavia, Italy \\ Correspondence: Department of Economics and Management, Via S. Felice, 5 - 27100 Pavia, Italy. E-mail: gior- \\ gio.giorgi@unipv.it
}

Received: February 3, 2017 Accepted: May 22, 2017 Online Published: July 25, 2017

doi:10.5539/jmr.v9n4p168 URL: https://doi.org/10.5539/jmr.v9n4p168

\begin{abstract}
We take into condideration necessary optimality conditions of minimum principle-type, that is for optimization problems having, besides the usual inequality and/or equality constraints, a set constraint. The first part pf the paper is concerned with scalar optimization problems; the second part of the paper deals with vector optimization problems.
\end{abstract}

Keywords: Minimum principle-type conditions, optimality conditions, set constraint, scalar optimization, vector optimization

AMS-2000 Math. Subj. Class. 90C29, 90C46, 90C30

\section{Introduction}

Minimum principle-type necessary optimality conditions usually occur in minimization problems where there is also a (not open) set constraint or abstract constraint, which takes into account those constraints that cannot be expressed by means of neither equalities nor inequalities. The name "minimum principle" necessary optimality criterion has been used by Mangasarian (1969) and has been referred to as a "maximum principle" in Halkin (1966), Halkin and Neustadt (1966), Canon, Cullum and Polak $(1966,1970)$ and in other authors, because of its relation to Pontryagin's maximum principle of optimal control theory. These minimum principle-type necessary optimality conditions may be considered extensions of the Fritz John-type necessary conditions and of the Kuhn-Tucker-type necessary conditions (when some constraint qualification is imposed) for scalar or vector optimization problems.

In the present paper we shall give an account of the main approaches adopted to study the said optimality conditions. We prefer to treat separately the scalar and the vector optimization problems, as the authors we take into consideration usually are concerned with only one type of the said problems. Obviously, several results established for scalar problems may be adapted to the vector case and, viceversa, several results obtained for vector problems may be easily "specialized" to the scalar case.

We perform our analysis in finite-dimensional spaces, under differentiability and continuous differentiability of the functions involved in the related problems. Minimum/maximum principle-type optimality conditions have been obtained also for problems in infinite-dimensional spaces; see, e. g., Jahn (1996, 2011), Kirsch and others (1978), Pshenichnyi (1971), Neustadt (1976), Ponstein (1980), Werner (1984).

We have no pretension to be exhaustive in our account: we take into consideration only some approaches that in our opinion have been significant for the study of the said problems. The paper is organized as follows.

In the present Section some basic tools and definitions (concerning mainly local cone approximations of sets) are recalled.

Section 2 is concerned with scalar optimization problems with only a set constraint, then with inequality constraints and a set constraint and finally with both inequality and equality constraints and a set constraint.

Section 3 deals with scalar problems, following the approach of Bertsekas and Ozdaglar (2002) who make use of the "general normal cone", introduced, among others, by Rockafellar (1993) and Rockafellar and Wets (1998).

Section 4 is concerned with vector optimization problems having, respectively, only a set constraint, inequality constraints and a set constraint, both inequality and equality constraints and a set constraint.

In Section 5 we recall few results of R. Cambini, taken from a series of papers of this author, concerning optimality conditions for general vector optimization problems having a set constraint.

Basic key tools in studying general optimality conditions in scalar and vector optimization problems are local cone ap- 
proximations of a set $X \subseteq \mathbb{R}^{n}$ at a point $x^{0}$ belonging to its closure. Let $X \subseteq \mathbb{R}^{n}$ be a nonempty set and let $\operatorname{cl}(X)$ be its closure; if $x^{0} \in \operatorname{cl}(X)$, then:

- the cone

$$
T\left(X, x^{0}\right)=\left\{\begin{array}{l}
v \in \mathbb{R}^{n}: \exists\left\{x^{k}\right\} \subseteq X, x^{k} \longrightarrow x^{0}, \exists\left\{\lambda_{k}\right\} \subseteq \mathbb{R}_{+}, \lambda_{k} \longrightarrow+\infty, \\
\text { such that } v=\lim _{k \longrightarrow+\infty} \lambda_{k}\left(x^{k}-x^{0}\right)
\end{array}\right\}
$$

is a closed cone, called Bouligand tangent cone to $X$ at $x^{0}$ or contingent cone to $X$ at $x^{0}$. Other representations of the contingent cone are:

$$
\begin{gathered}
T\left(X, x^{0}\right)=\left\{v \in \mathbb{R}^{n}: \forall U(v) \forall \lambda>0, \exists t \in(0, \lambda), \exists \bar{x} \in U(v) \text { such that } x^{0}+t \bar{x} \in X\right\} . \\
T\left(X, x^{0}\right)=\left\{\begin{array}{l}
v \in \mathbb{R}^{n}: \exists \varphi: \mathbb{R} \longrightarrow \mathbb{R}^{n} \text { with } \lim _{t \rightarrow 0^{+}} \varphi(t)=v, \forall \lambda>0, \\
\exists t \in(0, \lambda) \text { such that } x^{0}+t \varphi(t) \in X
\end{array}\right\} .
\end{gathered}
$$

It is well-known that the closed cone $T\left(X, x^{0}\right)$ is not necessarily convex. See, e. g., Aubin and Frankowska (1990), Bazaraa, Goode and Nashed (1974), Bazaraa and Shetty (1976), Giorgi and Guerraggio $(1992,2002)$ for other characterizations and for other remarks on the cone $T\left(X, x^{0}\right)$.

The closure of the convex hull of $T\left(X, x^{0}\right)$ is called the pseudotangent cone to $X$ at $x^{0}$ and denoted by $P\left(X, x^{0}\right)$ :

$$
P\left(X, x^{0}\right)=\operatorname{cl}\left(\operatorname{conv}\left(T\left(X, x^{0}\right)\right)\right)
$$

See Guignard (1969).

Other local cone approximations useful in optimization theory, and which are subcones of $T\left(X, x^{0}\right)$, are the following ones.

- The cone

$$
F\left(X, x^{0}\right)=\left\{v \in \mathbb{R}^{n}: \exists \lambda>0 \text { such that } \forall t \in(0, \lambda], x^{0}+t v \in X\right\}
$$

is called cone of feasible directions to $X$ at $x^{0}$ or cone of linear directions to $X$ at $x^{0}$.

- The cone

$$
I\left(X, x^{0}\right)=\left\{v \in \mathbb{R}^{n}: \exists U(v), \exists \lambda>0, \forall t \in(0, \lambda), \forall \bar{x} \in U(v) \text { we have } x^{0}+t \bar{x} \in X\right\}
$$

is an open cone, called cone of interior directions to $X$ at $x^{0}$ or inner tangent cone to $X$ at $x^{0}$. Another representation of this cone is the following one:

$$
I\left(X, x^{0}\right)=\left\{\begin{array}{l}
v \in \mathbb{R}^{n}: \forall \varphi: \mathbb{R} \longrightarrow \mathbb{R}^{n} \lim _{t \rightarrow 0^{+}} \varphi(t)=v, \exists \lambda>0, \\
\text { such that } \forall t \in(0, \lambda) \text { we have } x^{0}+t \varphi(t) \in X
\end{array}\right\} .
$$

It holds $x^{0} \in \operatorname{int}(X) \Longleftrightarrow 0 \in I\left(X, x^{0}\right)$.

- The cone

$$
Q\left(X, x^{0}\right)=\left\{\begin{array}{l}
v \in \mathbb{R}^{n}: \exists U(v), \forall \lambda>0 \exists t \in(0, \lambda) \text { such that } \forall \bar{x} \in U(v), \\
\text { we have } x^{0}+t \bar{x} \in X
\end{array}\right\}
$$

is an open cone, called cone of quasi-interior directions to $X$ at $x^{0}$ or also sequential interior tangent cone to $X$ at $x^{0}$. Another representation of this cone is the following one:

$$
Q\left(X, x^{0}\right)=\left\{\begin{array}{l}
v \in \mathbb{R}^{n}: \forall \lambda>0, \forall \varphi:(0, \lambda) \longrightarrow \mathbb{R}^{n}, \text { with } \lim _{t \rightarrow 0^{+}} \varphi(t)=v, \\
\exists t \in(0, \lambda) \text { such that } x^{0}+t \varphi(t) \in X
\end{array}\right\} .
$$

We have $x^{0} \in \operatorname{int}(X) \Longleftrightarrow 0 \in Q\left(X, x^{0}\right)$.

The structure of all the above local cone approximations depends only from a neighborhood of $x^{0}$ (they are "infinitesimal notions"). For eample, it holds

$$
T\left(X, x^{0}\right)=T\left(X \cap N\left(x^{0}\right), x^{0}\right),
$$

for any neighborhood $N\left(x^{0}\right)$. 
With reference to the cones introduced above, we have the following inclusions:

$$
\begin{aligned}
& I\left(X, x^{0}\right) \subseteq \quad Q\left(X, x^{0}\right) \subseteq T\left(X, x^{0}\right) \subseteq P\left(X, x^{0}\right) ; \\
& I\left(X, x^{0}\right) \subseteq F\left(X, x^{0}\right) \subseteq T\left(X, x^{0}\right) \subseteq P\left(X, x^{0}\right) .
\end{aligned}
$$

None of the cones introduced above are in general convex. But if we assume $X$ to be a convex set, we obtain the convexity of the approximating cones. In effect, it is sufficient that $X$ is locally convex at $x^{0}$, that is there exists an arbitrary neighborhood $N\left(x^{0}\right)$ such that $X \cap N\left(x^{0}\right)$ is convex. We have the following results:

Let $X \subseteq \mathbb{R}^{n}$ be a convex set or even a locally convex set at $x^{0} \in \operatorname{cl}(X)$. Then the cones $F\left(X, x^{0}\right), I\left(X, x^{0}\right), Q\left(X, x^{0}\right)$ and $T\left(X, x^{0}\right)$ are convex and it holds

$$
\begin{gathered}
I\left(X, x^{0}\right)=Q\left(X, x^{0}\right)=\operatorname{cone}\left(\operatorname{int}(X), x^{0}\right) \\
\operatorname{cl}\left(F\left(X, x^{0}\right)\right)=\operatorname{cl}\left(\operatorname{cone}\left(X, x^{0}\right)\right) \\
P\left(T, x^{0}\right)=T\left(X, x^{0}\right)=\operatorname{cl}\left(\operatorname{cone}\left(X, x^{0}\right)\right)=\operatorname{cl}\left(F\left(X, x^{0}\right)\right)
\end{gathered}
$$

where

$$
\operatorname{cone}\left(X, x^{0}\right)=\left\{v \in \mathbb{R}^{n}: v=\lambda\left(x-x^{0}\right), \lambda>0, x \in X\right\}
$$

is the cone generated by $X-x^{0}$. This cone is also denoted by $\mathbb{R}_{+}\left(X-x^{0}\right)$.

If, moreover, $\operatorname{int}(X) \neq \varnothing$, then

$$
\begin{aligned}
I\left(X, x^{0}\right) & =\operatorname{int}\left(T\left(X, x^{0}\right)\right) ; \\
T\left(X, x^{0}\right) & =\operatorname{cl}\left(I\left(X, x^{0}\right)\right) .
\end{aligned}
$$

If $X$ is polyhedral, then

$$
F\left(X, x^{0}\right)=T\left(X, x^{0}\right)
$$

We recall that, given a set $S \subseteq \mathbb{R}^{n}$, the (negative) polar cone $S^{*}$ of $S$ is given by

$$
S^{*}=\left\{v \in \mathbb{R}^{n}: v x \leqq 0, \forall x \in S\right\}
$$

It is well-known that if $S$ is a non-empty closed convex cone, then $S=\left(S^{*}\right)^{*}$. If $S$ is a linear subspace, then $S^{*}$ is nothing else that $S^{\perp}$.

\section{Scalar Optimization with a Set Constraint. Basic Results}

In the present section we shall consider the followinh nonlinear programming problems.

$$
\begin{aligned}
(P)_{1}: & \min f(x), \quad x \in X \\
(P)_{2}: & \left\{\begin{array}{l}
\min f(x) \\
g_{i}(x) \leqq 0, \quad i=1, \ldots, m \\
x \in X
\end{array}\right. \\
(P)_{3}: & \left\{\begin{array}{l}
\min f(x) \\
g_{i}(x) \leqq 0, \quad i=1, \ldots, m \\
h_{j}(x)=0, j=1, \ldots, r<n, \\
x \in X
\end{array}\right.
\end{aligned}
$$

where $X$ is a subset (not necessarily open) of $\mathbb{R}^{n}, f: \Omega \longrightarrow \mathbb{R}$ and every $g_{i}: \Omega \longrightarrow \mathbb{R}, i=1, \ldots, m$, are differentiable on an open set $\Omega \subseteq \mathbb{R}^{n}$ containing the feasible set of the above problems, and every $h_{j}: \Omega \longrightarrow \mathbb{R}, j=1, \ldots, r<n$, is continuously differentiable on $\Omega$ (it is obviously possible to assume less stringent differentiability conditions).

With regard to problem $(P)_{1}$ the following result, due to Gould and Tolle (1971), Guignard (1969), Hestenes (1966, 1975), Varaiya (1967), is of basic importance.

Theorem 1. If $x^{0}$ is a local solution of $(P)_{1}$, then

$$
-\nabla f\left(x^{0}\right) \in\left(T\left(X, x^{0}\right)\right)^{*}
$$


Guignard (1969) gives the following formulation of the thesis of Theorem 1:

$$
-\nabla f\left(x^{0}\right) \in\left(P\left(X, x^{0}\right)\right)^{*} .
$$

However, as it is true that for any nonempty set $C \subseteq \mathbb{R}^{n}$, it holds $C^{*}=(\operatorname{cl}(\operatorname{conv}(C)))^{*}$, we obtain $\left(T\left(X, x^{0}\right)\right)^{*}=\left(P\left(X, x^{0}\right)\right)^{*}$. If the set $X$ is convex, then we have $T\left(X, x^{0}\right)=\operatorname{cl}\left(\operatorname{cone}\left(X, x^{0}\right)\right)$ and it is easy to see that a vector $v$ belongs to $\left(T\left(X, x^{0}\right)\right)^{*}$ if and only if

$$
v\left(x-x^{0}\right) \leqq 0, \quad \forall x \in X .
$$

A vector $v$ satisfying (2), with $X$ a convex set, is said to be normal to $X$ at $x^{0} \in X$. The set of all normals to $X$ (convex) at $x^{0}$ is called normal cone to $X$ at $x^{0}$ and is denoted by $N\left(X, x^{0}\right)$ :

$$
N\left(X, x^{0}\right)=\left\{v \in \mathbb{R}^{n}: v\left(x-x^{0}\right) \leqq 0, \quad \forall x \in X\right\},
$$

i.e.

$$
N\left(X, x^{0}\right)=\left[\operatorname{cone}\left(X-x^{0}\right)\right]^{*} .
$$

Therefore when $X$ is a convex set, (1) becomes

$$
-\nabla f\left(x^{0}\right) \in N\left(X, x^{0}\right)
$$

or

$$
0 \in \nabla f\left(x^{0}\right)+N\left(X, x^{0}\right)
$$

or also

$$
\nabla f\left(x^{0}\right)\left(x-x^{0}\right) \geqq 0, \quad \forall x \in X .
$$

This necessary condition becomes also sufficient (for the global optimality of $x^{0}$ in $\left.(P)_{1}\right)$ if $f$ is convex or psudoconvex (see, e. g., Mangasarian (1969)). We note moreover, that the Bouligand tangent cone and the normal cone at a point $x^{0} \in X$ of the convex subset $X$ of $\mathbb{R}^{n}$ are polar cones of each other, i. e.

$$
\begin{aligned}
& T\left(X, x^{0}\right)=\left\{v \in \mathbb{R}^{n}: v y \leqq 0, \quad \forall y \in N\left(X, x^{0}\right)\right\}, \\
& N\left(X, x^{0}\right)=\left\{y \in \mathbb{R}^{n}: y v \leqq 0, \quad \forall v \in T\left(X, x^{0}\right)\right\} .
\end{aligned}
$$

Of course if $x^{0} \in \operatorname{int}(X)$, it holds $T\left(X, x^{0}=\mathbb{R}^{n},\left(T\left(X, x^{0}\right)\right)^{*}=\{0\}\right.$ and Theorem 1 recovers the classical Fermat's necessary condition for an unconstrained local minimum.

Let us now consider problem $(P)_{2}$. If we define the sets $A=\left\{x \in \mathbb{R}^{n}: g_{i}(x) \leqq 0, I=1, \ldots, m\right\}$ and $S=X \cap A$, we have, under the same assumptions of Theorem 1, that if $x^{0}$ is a local solution of $(P)_{2}$, then

$$
-\nabla f\left(x^{0}\right) \in\left(T\left(S, x^{0}\right)\right)^{*} .
$$

Let us denote by

$$
I\left(x^{0}\right)=\left\{i: g_{i}\left(x^{0}\right)=0\right\}
$$

the set of the active constraints at $x^{0}$ for $(P)_{2}$ and by

$$
G_{0}=\left\{v \in \mathbb{R}^{n}: \nabla g_{i}\left(x^{0}\right) v \leqq 0, \quad \forall i \in I\left(x^{0}\right)\right\}
$$

the linearizing cone at $x^{0}$ for $(P)_{2}$. It is well-known that it holds

$$
T\left(S, x^{0}\right) \subseteq G_{0},
$$

i. e.

$$
\left(G_{0}\right)^{*} \subseteq\left(T\left(S, x^{0}\right)\right)^{*}
$$

As $\left(T\left(S, x^{0}\right)\right)^{*}$ is a (closed) convex cone, from

$$
\left(T\left(S, x^{0}\right)\right)^{*}=\left(G_{0}\right)^{*} \cup\left(\left(T\left(S, x^{0}\right)\right)^{*} \backslash\left(G_{0}\right)^{*} \cup\left\{x^{0}\right\}\right)
$$


we obtain

$$
\left(T\left(S, x^{0}\right)\right)^{*}=\left(G_{0}\right)^{*}+\left(\left(T\left(S, x^{0}\right)\right)^{*} \backslash\left(G_{0}\right)^{*} \cup\left\{x^{0}\right\}\right),
$$

that is, thanks to Farkas' lemma,

$$
\left(T\left(S, x^{0}\right)\right)^{*}=\Gamma_{0}+\left(\left(T\left(S, x^{0}\right)\right)^{*} \backslash\left(G_{0}\right)^{*} \cup\left\{x^{0}\right\}\right),
$$

where

$$
\Gamma_{0}=\left\{v \in \mathbb{R}^{n}: v=\sum_{i \in I\left(x^{0}\right)} \lambda_{i} \nabla g_{i}\left(x^{0}\right), \quad \lambda_{i} \geqq 0, \quad \forall i \in I\left(x^{0}\right)\right\},
$$

is the so-called cone of gradients at $x^{0}$.

We have therefore the following result, due to Gould and Tolle (1972).

Theorem 2. If $x^{0}$ is a local solution of $(P)_{2}$, then there exist scalars $\lambda_{i} \geqq 0, i \in I\left(x^{0}\right)$, such that

$$
-\left(\nabla f\left(x^{0}\right)+\sum_{i \in I\left(x^{0}\right)} \lambda_{i} \nabla g_{i}\left(x^{0}\right)\right) \in\left(\left(T\left(S, x^{0}\right)\right)^{*} \backslash\left(G_{0}\right)^{*} \cup\left\{x^{0}\right\}\right) .
$$

On the previous theorem the following remarks can be useful.

Remark 1. If it holds

$$
\left(G_{0}\right)^{*}=\left(T\left(S, x^{0}\right)\right)^{*}
$$

obviously relation (3) becomes

$$
\nabla f\left(x^{0}\right)+\sum_{i \in I\left(x^{0}\right)} \lambda_{i} \nabla g_{i}\left(x^{0}\right)=0
$$

Relation (4) is a well-known constraint qualification for problem $(P)_{2}$ and it was proved by Gould and Tolle (1971) that this condition is both necessary and sufficient for the so-called "Lagrangian regularity" for $(P)_{2}$, i. e. for the validity of (5) with respect to any differentiable objective function, having a local constrained minimum at $x^{0} \in S$. When condition (4) is not satisfied, Theorem 2 gives a sort of generalization of the classical Karush-Kuhn-Tucker conditions. The fact that in (3) the multiplier of $\nabla f\left(x^{0}\right)$ is just the unity, is not in contrast with what specified in the classical Fritz John conditions, as the cone $\left(\left(T\left(S, x^{0}\right)\right)^{*} \backslash\left(G_{0}\right)^{*} \cup\left\{x^{0}\right\}\right)$ does not vecessarily shrink to $\{0\}$ if $x^{0} \in \operatorname{int}(X)$.

The last lines of the previous remark induce to find necessary optimality conditions for $(P)_{2}$, expressed by the belonging of the negative of the gradient of the Lagrangian function of $(P)_{2}$ to the cone $\left(T\left(X, x^{0}\right)\right)^{*}$. The next result is due to Gould and Tolle (1972).

Theorem 3. Let $x^{0}$ be a local solution of $(P)_{2}$ and let the following condition be satisfied:

$$
\left(\left(T\left(S, x^{0}\right)\right)^{*}=\left(G_{0}\right)^{*}+\left(T\left(X, x^{0}\right)\right)^{*} .\right.
$$

Then, there exist scalars $\lambda_{i} \geqq 0, i \in I\left(x^{0}\right)$, such that

$$
-\left(\nabla f\left(x^{0}\right)+\sum_{i \in I\left(x^{0}\right)} \lambda_{i} \nabla g_{i}\left(x^{0}\right)\right) \in\left(T\left(X, x^{0}\right)\right)^{*} .
$$

The same authors prove that condition (6) is both necessary and sufficient for the validity of (7) with respect to any differentiable objective function $f$ having a local minimum at $x^{0} \in S$. More generally (see Guignard (1969)), we have also the following optimality condition for $(P)_{2}$. Suppose that $K$ is a closed convex cone in $\mathbb{R}^{n}$ satisfying the assumptions

a) $G_{0} \cap K=P\left(S, x^{0}\right)$;

b) $\left(G_{0}\right)^{*}+K^{*}$ is closed.

If $x^{0}$ is a local solution of $(P)_{2}$, then there exist multipliers $\lambda_{i} \geqq 0, i \in I\left(x^{0}\right)$, such that

$$
-\left(\nabla f\left(x^{0}\right)+\sum_{i \in I\left(x^{0}\right)} \lambda_{i} \nabla g_{i}\left(x^{0}\right)\right) \in K^{*} .
$$

We remark that if $K$ is a polyhedral cone, then assumption b) is surely satisfied, as $G_{0}$ is clearly a polyhedral cone. If $\operatorname{relint}\left(G_{0}\right) \cap \operatorname{relint}(K) \neq \varnothing$, the above assunmption b) holds. 
The next result is a Fritz John-type optimality condition (or minimum principle-type optimality condition) for $(P)_{2}$ and was proved by Giorgi and Guerraggio (1994).

Theorem 4. Let $x^{0}$ be a local solution of $(P)_{2}$. Then, there exist scalars $\lambda_{0} \geqq 0, \lambda_{i} \geqq 0, i \in I\left(x^{0}\right)$, not all zero, such that

$$
-\left(\lambda_{0} \nabla f\left(x^{0}\right)+\sum_{i \in I\left(x^{0}\right)} \lambda_{i} \nabla g_{i}\left(x^{0}\right)\right) \in\left(T_{1}\left(X, x^{0}\right)\right)^{*}
$$

where $T_{1}\left(X, x^{0}\right)$ is any convex subcone of $T\left(X, x^{0}\right)$, with vertex at $0 \in T_{1}\left(X, x^{0}\right)$.

Remark 2. If (6) holds, then in (8) we have $\lambda_{0}=1$ and $T_{1}\left(X, x^{0}\right)$ can be chosen equal to $T\left(X, x^{0}\right)$. If $T\left(X, x^{0}\right)$ is a convex cone, then (8) gives a sharper result, as $T_{1}\left(X, x^{0}\right)$ can be chosen equal to $T\left(X, x^{0}\right)$. If $T\left(X, x^{0}\right)$ is not a convex cone, there exist some convex subcones of the same, that can be chosen to represent $T_{1}\left(X, x^{0}\right)$ in (8). It is well-known that one of these convex subcones of $T\left(X, x^{0}\right)$ is the Clarke tangent cone (see the next Section and see, e. g., Giorgi and Guerraggio (1992), Ward (1987)). Obviously, if we can choose the largest convex subcone of $T\left(X, x^{0}\right)$, Theorem 4 will be sharper; a convex subcone of $T\left(X, x^{0}\right)$ larger than the Clarke tangent cone is the Michel-Penot proto-tangent cone (see Michel and Penot (1984), Ward (1987) and also Martin and Watkins (1985), where the expression "external cone" is used). Treiman (1991) has shown that there are infinite convex cones lying between the Clarke tangent cone and the Michel-Penot prototangent cone. Therefore we have the possibility to choose all the convex subcones of $T\left(X, x^{0}\right)$ we wish. For the reader's convenience we quote also the results of Nagahisa and Sakawa (1969) who reformulate Theorem 4, replacing $T_{1}\left(X, x^{0}\right)$ with an arbitrary convex set $K \subseteq \mathbb{R}^{n}$ such that

$$
0 \in K, \quad K \subseteq T\left(X, x^{0}\right) .
$$

Other considerations on $(P)_{2}$ are given in the recent papers of Flores-Bazan (2014) and Flores-Bazan and Mastroeni (2015).

If $X$ is convex (or locally convex at $x^{0} \in X$ ), then (8) can be expressed in the form

$$
-\left(\lambda_{0} \nabla f\left(x^{0}\right)+\sum_{i \in I\left(x^{0}\right)} \lambda_{i} \nabla g_{i}\left(x^{0}\right)\right)\left(x-x^{0}\right) \geqq 0, \quad \forall x \in X,
$$

with $\lambda_{0} \geqq 0, \lambda_{i} \geqq 0, i \in I\left(x^{0}\right)$, not all zero. Perhaps more usually, relation (9) is written in the form

$$
0 \in \lambda_{0} \nabla f\left(x^{0}\right)+\sum_{i \in I\left(x^{0}\right)} \lambda_{i} \nabla g_{i}\left(x^{0}\right)+N\left(X, x^{0}\right)
$$

where $N\left(X, x^{0}\right)$ is the normal cone to $X$ at $x^{0} \in X$. A sufficient condition to have in (9) $\lambda_{0}>0$ is: for some $\hat{x} \in X$ it holds

$$
\nabla g_{i}\left(x^{0}\right)\left(\hat{x}-x^{0}\right)<0, \quad \forall i \in I\left(x^{0}\right) .
$$

We now consider $(P)_{3}$. In the usual classical treatments of the Fritz John necessary conditions for $(P)_{3}$, the set $X$ is assumed to be open or that the optimal point $x^{0}$ belongs to $\operatorname{int}(X)$; of course this last assumption can be verified if $\operatorname{int}(X) \neq \varnothing$. See, e. g., Mangasarian and Fromovitz (1967). When none of the said assumptions is verified, it is no longer assured that $x^{0}$ is a stationary point for the associated (augmented) Lagrangian function of $(P)_{3}$, similarly to what said for $(P)_{2}$. See the counterexample of Bazaraa and Goode (1972) and Bazaraa and Shetty (1976). Mangasarian (1969) treats the case of $X$ convex and assumes that $\operatorname{int}(X) \neq \varnothing$. This author then proves the following result.

Theorem 5. Let $X$ be a convex set, with $\operatorname{int}(X) \neq \varnothing$; let $x^{0}$ be a local solution of $(P)_{3}$. Then there exist multipliers $\lambda_{0}, \lambda_{i}$, $i=1, \ldots, m ; \mu_{j}, j=1, \ldots, r$, such that

a) $\left(\lambda_{0} \nabla f\left(x^{0}\right)+\sum_{i=1}^{m} \lambda_{i} \nabla g_{i}\left(x^{0}\right)+\sum_{j=1}^{r} \mu_{j} \nabla h_{j}\left(x^{0}\right)\right)\left(x-x^{0}\right) \geqq 0, \forall x \in X$,

b) $\lambda_{i} g_{i}\left(x^{0}\right)=0, i=1, \ldots, m$,

c) $\quad \lambda_{0} \geqq 0, \lambda_{i} \geqq 0, i=1, \ldots, m,\left(\lambda_{0}, \lambda_{1}, \ldots, \lambda_{m}, \mu_{1}, \ldots, \mu_{r}\right) \neq 0$.

Mangasarian (1969, p. 169) remarks that the assumption $\operatorname{int}(X) \neq \varnothing$ "is a mild restriction, since in general a convex set without an interior is equivalent to the intersection of a convex set with a nonempty interior and a linear manifold

$$
\left\{x: x \in \mathbb{R}^{n}, h(x)=0\right\},
$$

$h$ linear on $\mathbb{R}^{n}$ ". However, this author does not further develop this remark. 
An equivalent expression of relation a) of Theorem 5 is

$$
0 \in \lambda_{0} \nabla f\left(x^{0}\right)+\sum_{i=1}^{m} \lambda_{i} \nabla g_{i}\left(x^{0}\right)+\sum_{j=1}^{r} \mu_{j} \nabla h_{j}\left(x^{0}\right)+N\left(X, x^{0}\right) .
$$

Always with reference to $(P)_{3}$ it must be noted that Theorem 2 can be immediately fitted to $(P)_{3}$; let us assume the following notations:

$$
\begin{gathered}
A=\left\{x \in \mathbb{R}^{n}: g_{i}(x) \leqq 0, i=1, \ldots, m\right\} ; \\
B=\left\{x \in \mathbb{R}^{n}: h_{j}(x)=0, j=1, \ldots, r\right\} ; \\
S_{1}=X \cap A \cap B=X \cap C, \text { where } C=A \cap B . \\
H_{0}=\operatorname{ker}\left(\nabla h\left(x^{0}\right)\right)=\left\{v \in \mathbb{R}^{n}: \nabla h_{j}\left(x^{0}\right) v=0, \forall j=1, \ldots, r\right\}, \\
E_{0}=G_{0} \cap H_{0} .
\end{gathered}
$$

Theorem 6. Let $x^{0}$ be a local solution of $(P)_{3}$; then there exist multipliers $\lambda_{i} \geqq 0, i \in I\left(x^{0}\right), \mu_{j} \in \mathbb{R}, j=1, \ldots, r$, such that

$$
\nabla f\left(x^{0}\right)+\sum_{i \in I\left(x^{0}\right)} \lambda_{i} \nabla g_{i}\left(x^{0}\right)+\sum_{j=1}^{r} \mu_{j} \nabla h_{j}\left(x^{0}\right) \in\left(T\left(S_{1}, x^{0}\right)\right)^{*} \backslash E_{0}^{*} \cup\{0\} .
$$

Bazaraa and Goode (1972) have obtained for $(P)_{3}$ a version similar to the optimality conditions for $(P)_{2}$, given in Theorem 4. However, the presence in $(P)_{3}$ of equality constraints, does not allow to use a closed cone, such as the Bouligand tangent cone. The said authors use the cone of interior directions at $x^{0} \in X$, which is an open cone. They prove the following result.

Theorem 7. Let $x^{0}$ be a local solution of $(P)_{3}$ and let the cone of interior directions $I\left(X, x^{0}\right)$ be convex. Then, there exist multipliers $\lambda_{0} \geqq 0, \lambda_{i} \geqq 0, i \in I\left(x^{0}\right), \mu_{j} \in \mathbb{R}, j=1, \ldots, r$, with $\left(\lambda_{0}, \lambda_{i}, \mu_{j}\right) \neq 0$, such that

$$
-\left(\lambda_{0} \nabla f\left(x^{0}\right)+\sum_{i \in I\left(x^{0}\right)} \lambda_{i} \nabla g_{i}\left(x^{0}\right)+\sum_{j=1}^{r} \mu_{j} \nabla h_{j}\left(x^{0}\right)\right) \in\left(I\left(X, x^{0}\right)\right)^{*} .
$$

Theorem 7 has been sharpened by Giorgi and Guerraggio (1994) who obtain an optimality condition for $(P)_{3}$, similar to (10), but with the cone of quasi-interior directions $Q\left(X, x^{0}\right)$ replacing the cone $I\left(X, x^{0}\right)$; indeed it holds, as previously remarked, $I\left(X, x^{0}\right) \subseteq Q\left(X, x^{0}\right)$. A result similar to Theorem 7 has been established by Pshenichnyi (1971) and by Pshenichnyi and Danilin (1978). Canon, Cullum and Polak $(1969,1970)$ give necessary conditions of optimality for a mathematical programming problem with a set constraint and equality constraints only, by means of rather complicate definitions of local cone approximations: the linearization of the first kind and the linearization of the second kind of the constraint set $X$. For interesting considerations on these approximation cones the reader is referred to Martin, Gardner and Watkins (1981). Halkin and Neustadt (1966) and Neustadt $(1969,1976)$ give a general multiplier rule in an infinite-dimensional setting by means of particular "differentiability" assumptions on the functions involved in their problem and by means of a rather complicate approximation of the constraint set. Similar comments can be made for the paper of Nagahisa (1988).

Remark 3. When $X$ is convex and $\operatorname{int}(X) \neq \varnothing$, Theorem 7 recovers Theorem 5 of Mangasarian (1969). Indeed, in this case it holds

$$
T\left(X, x^{0}\right)=\operatorname{cl}\left(I\left(X, x^{0}\right)\right)=\operatorname{cl}\left(Q\left(X, x^{0}\right)\right)=\operatorname{cl}\left(\operatorname{cone}\left(X, x^{0}\right)\right) .
$$

If, moreover, there exists $\hat{x} \in \operatorname{int}(X)$ or even $\hat{x} \in \operatorname{relint}(X)$ such that

$$
\begin{aligned}
\nabla g_{i}\left(x^{0}\right)\left(\hat{x}-x^{0}\right) & <0, \forall i \in I\left(x^{0}\right), \\
\nabla h_{j}\left(x^{0}\right)\left(\hat{x}-x^{0}\right) & =0, \forall j=1, \ldots, r
\end{aligned}
$$

and $\nabla h_{j}\left(x^{0}\right), j=1, \ldots, r$, are linearly independent, then in relation a) of Theorem 5 we can take $\lambda_{0}=1$. This one is a slight generalization of the well-known Mangasarian-Fromovitz constraint qualification (see Mangasarian and Fromovitz (1967), Mangasarian (1969)).

If $X$ is convex with $\operatorname{int}(X)=\varnothing$, then $I\left(X, x^{0}\right)$ and $Q\left(X, x^{0}\right)$ are empty and therefore $\left(I\left(X, x^{0}\right)\right)^{*}=\left(Q\left(X, x^{0}\right)\right)^{*}=\mathbb{R}^{n}$ and hence Theorem 7 is of no utility. However, several authors (see, e. g., Bertsekas and Ozdaglar (2002), Jimenez and Novo (2002a), Giorgi, Jimenez and Novo (2004), Robinson (1976)) have shown that condition $\operatorname{int}(X) \neq \varnothing$, with $X$ convex, can be dispensed, in order to obtain Theorem 5. See the next Section and the Sections concerning vector optimization. 
Remark 4. Similarly to what previously remarked for $(P)_{2}$, the set $S_{1}$ is said to be Lagrange regular at $x^{0} \in S_{1}$ if, for every differentiable objective function $f$ having a constrained local minimum at $x^{0}$, relation (10) holds with $\lambda_{0}=1$. Bazaraa and Goode (1972) have shown that the following constraint qualification

$$
\left(T\left(S_{1}, x^{0}\right)\right)^{*}=\left(I\left(X, x^{0}\right)\right)^{*}+\left(G_{0}\right)^{*}+\left(H_{0}\right)^{*}
$$

is both necessary and sufficient for the Lagrange regularity of $S_{1}$ at $x^{0} \in S_{1}$. Finally, if the Guignard-Gould-Tolle constraint qualification is imposed to hold at $x^{0} \in S_{1}$, i. e. if

$$
\left(T\left(S_{1}, x^{0}\right)\right)^{*}=\left(G_{0} \cap H_{0}\right)^{*},
$$

then (10) becomes the classical relation:

$$
\nabla f\left(x^{0}\right)+\sum_{i \in I\left(x^{0}\right)} \lambda_{i} \nabla g_{i}\left(x^{0}\right)+\sum_{j=1}^{r} \mu_{j} \nabla h_{j}\left(x^{0}\right)=0 .
$$

It is well-known that the Guignard-Gould-Tolle constraint qualification is both necessary and suffcient for relation (11) to hold (together with the non-negativity of the multipliers and with the complementarity conditions) for any differentiable objective function having a local minimum at $x^{0} \in S_{1}$. See Gould and Tolle (1971).

Di and Poliquin (1994) have relaxed the usual assumptions of continuous differentiability of functions $h_{j}, j=1, \ldots, r$, imposed by several authors to obtain optimality condition (11). Other considerations on $(P)_{3}$ along similar lines of Di and Poliquin, are made in Di (1996).

\section{Scalar Optimization with a Set Constraint: the Approach by Means of General Normal Cones}

Besides the classical normal cone of the Convex Analysis, recalled in Section 2, there are other notions of normal cone, useful in nonsmooth analysis, optimization theory, variational analysis, etc. In the present Section we consider a set $X \subseteq \mathbb{R}^{n}$ to be a closed set and a point $x^{0} \in X$. The general normal cone or Mordukhovich normal cone to $X$ at $x^{0}$ (see Mordukhovich (1976), Rockafellar (1993), Rockafellar and Wets (1998)) consists of all vectors $v \in \mathbb{R}^{n}$ for which there is a sequence of vectors $v^{k} \longrightarrow v$ and a sequence of points $x^{k} \longrightarrow x^{0}$ in $X$, such that, for each $k$,

$$
\left(v^{k}\left(x-x^{k}\right)\right) \leqq o\left(\left\|x-x^{k}\right\|\right), \forall x \in X .
$$

The general normal cone will be denoted by $N^{g}\left(X, x^{0}\right)$. Rockafellar and Wets (1998) prove that $v \in N^{g}\left(X, x^{0}\right)$ if and only if there exist sequences $\left\{x^{k}\right\} \subseteq X$ and $\left\{v^{k}\right\}$, such that $x^{k} \longrightarrow x^{0}, v^{k} \longrightarrow v$ and $v^{k} \in\left(T\left(X, x^{0}\right)\right)^{*}$ for all $k . N^{g}\left(X, x^{0}\right)$ is a closed cone, but in general is not convex. If, in relation (12) the sequences can be chosen constant, i. e.

$$
\left(v\left(x-x^{0}\right)\right) \leqq o\left(\left\|x-x^{0}\right\|\right), \forall x \in X,
$$

we obtain the regular normal cone to $X$ at $x^{0}$, denoted $N^{r}\left(X, x^{0}\right)$ and also called (Mordukhovich (2005)) Fréchet normal cone to $X$ at $x^{0}$.

$N^{r}\left(X, x^{0}\right)$ is a closed convex cone. In general it holds

$$
\left(T\left(X, x^{0}\right)\right)^{*} \subseteq N^{g}\left(X, x^{0}\right)
$$

but

$$
\left(T\left(X, x^{0}\right)\right)^{*}=N^{r}\left(X, x^{0}\right) .
$$

When relation (13) holds with an equality sign, then set $X$ is called "regular" at $x^{0} \in X$. A normal vector $v$ to $X$ at $x^{0}$ is then called regular normal vector, i. e. it holds $N^{g}\left(X, x^{0}\right)=N^{r}\left(X, x^{0}\right)$, or

$$
v \in N^{r}\left(X, x^{0}\right) \Longleftrightarrow v y \leqq 0, \text { for all } y \in T\left(X, x^{0}\right) .
$$

If $X$ is convex, then $X$ is regular at each $x \in X$ and it holds

$$
N^{g}\left(X, x^{0}\right)=N^{r}\left(X, x^{0}\right)=N\left(X, x^{0}\right)=\left\{v \in \mathbb{R}^{n}: v\left(x-x^{0}\right) \leqq 0, \forall x \in X\right\} .
$$

If $X$ is regular at some $x \in X$, then $T(X, x)$ is convex and the cones $T(X, x)$ and $N^{r}(X, x)$ are polar to each other.

Moreover, Rockafellar (1993) and Rockafellar and Wets (1998) prove the following interesting proposition which characterizes the gradients of regular normals. 
Theorem 8. For any closed set $X \subseteq \mathbb{R}^{n}$ the following properties of a vector $v \in \mathbb{R}^{n}$ are equivalent:

a) $\quad v$ is a regular normal to $X$ at $x^{0} \in X$.

b) $v \in\left(T\left(X, x^{0}\right)\right)^{*}$.

c) On some neighborhood $U\left(x^{0}\right)$ of $x^{0}$ there is a smooth function $f$, with $-\nabla f\left(x^{0}\right)=v$, such that $f$ attains its minimum relative to $X \cap U\left(x^{0}\right)$ at $x^{0}$.

Bertsekas and Ozdaglar (2002) consider problem $(P)_{3}$, with $X$ a nonempty closed set of $\mathbb{R}^{n}$ and with $f$, every $g_{i}$ and every $h_{j}$ continuously differentiable functions ( $C^{1}$-functions) from $\mathbb{R}^{n}$ to $\mathbb{R}$. They prove the following interesting "enhanced Fritz John conditions".

Theorem 9. Let $x^{0}$ be a local solution of $(P)_{3}$, where $X$ is a nonempty closed set of $\mathbb{R}^{n}$. Then, there exist scalars $\lambda_{0}, \lambda_{1}, \ldots, \lambda_{m}$ and $\mu_{1}, \ldots, \mu_{r}$ satisfying the following conditions:

i) $\quad-\left[\lambda_{0} \nabla f\left(x^{0}\right)+\sum_{i=1}^{m} \lambda_{i} \nabla g_{i}\left(x^{0}\right)+\sum_{j=1}^{r} \mu_{j} \nabla h_{j}\left(x^{0}\right)\right] \in N^{g}\left(X, x^{0}\right)$;

ii) $\quad \lambda_{i} \geqq 0$ for all $i=0,1, \ldots, m$;

iii) $\lambda_{0}, \lambda_{1}, \ldots, \lambda_{m}, \mu_{1}, \ldots, \mu_{r}$ are not all equal to zero;

iv) if the index set $I \cup J$ is nonempty, where $I=\left\{i: i \neq 0, \lambda_{i}>0\right\} ; \quad J=\left\{j: \mu_{j} \neq 0\right\}$, there exists a sequence $\left\{x^{k}\right\} \subseteq X$ that converges to $x^{0}$ and is such that for all $k$, $f\left(x^{k}\right)<f\left(x^{0}\right), \mu_{j} h_{j}\left(x^{k}\right)>0, \forall j \in J, \lambda_{i} g_{i}\left(x^{k}\right)>0, \forall i \in I, g_{i}^{+}\left(x^{k}\right)=o\left(w\left(x^{k}\right)\right), \forall i \notin I ;$ $\left|h_{j}\left(x^{k}\right)\right|=o\left(w\left(x^{k}\right)\right), \forall j \notin J$, where

$$
w(x)=\min \left\{\min _{i \in I} g_{i}^{+}(x), \min _{j \in J}\left|h_{j}(x)\right|\right\}
$$

and

$$
g_{i}^{+}(x)=\max \left\{0, g_{i}(x)\right\} .
$$

Following Bertsekas and Ozdaglar, we point out that condition $i v$ ) of the previous theorem is stronger than the usual complementarity conditions. We note moreover that when $X$ is a convex set, relation $i$ ) collapses to relation a) of Theorem 5 , and this without requiring that $\operatorname{int}(X) \neq \varnothing$.

On the other hand, Clarke (1976) presented the following generalized Lagrangian multipliers rule for $(P)_{3}$, where $f$, every $g_{i}$ and every $h_{j}$ are supposed to be locally Lipschitz at the feasible point $x^{0}$ and that $X$ is a closed subset of $\mathbb{R}^{n}$.

Theorem 10. Let $x^{0}$ be a local solution of $(P)_{3}$; then there exist multipliers $r_{0}, r_{i}, s_{j}(i=1, \ldots, m ; j=1, \ldots, r)$, not all zero, such that

a) $r_{0} \geqq 0, r_{i} \geqq 0, i=1, \ldots, m$;

b) $r_{i} g_{i}\left(x^{0}\right)=0, i=1, \ldots, m$;

c) $\quad-\left[r_{0} \partial^{C} f\left(x^{0}\right)+\sum_{i=1}^{m} r_{i} \partial^{C} g_{i}\left(x^{0}\right)+\sum_{j=1}^{r} s_{j} \partial^{C} h_{j}\left(x^{0}\right)\right] \in N^{C}\left(X, x^{0}\right)$.

In the previous theorem $\partial^{C} f\left(x^{0}\right)$ is the Clarke subdifferential of $f$ at $x^{0}$, i. e.

$$
\partial^{C} f\left(x^{0}\right)=\left\{\xi \in \mathbb{R}^{n}: f^{\circ}\left(x^{0}, v\right) \geqq \xi^{\top} v, \forall v \in \mathbb{R}^{n}\right\},
$$

where

$$
f^{\circ}\left(x^{0}, v\right)=\limsup _{y \rightarrow x^{0}, \lambda \downarrow 0} \lambda^{-1}[f(y+\lambda v)-f(y)]
$$

is the Clarke directional derivative at $x^{0} \in \Omega$ in the direction $v$. It is well-known that $\partial^{C} f\left(x^{0}\right)$ is a nonempty convex compact subset of $\mathbb{R}^{n}$ and that

$$
f^{\circ}\left(x^{0}, v\right)=\max \left\{\xi^{\top} v: \xi \in \partial^{C} f\left(x^{0}\right)\right\} .
$$

Similarly for $\partial^{C} g_{i}\left(x^{0}\right)$ and $\partial^{C} h_{j}\left(x^{0}\right)$. The cone $N^{C}\left(X, x^{0}\right)$ is the Clarke normal cone to $X$ at $x^{0} \in X$ (see Clarke (1983)), i. e.

$$
N^{C}\left(X, x^{0}\right)=\left(T^{C}\left(X, x^{0}\right)\right)^{*},
$$

where $T^{C}\left(X, x^{0}\right)$ is the Clarke tangent cone to $X$ at $x^{0} \in X$ :

$$
T^{C}\left(X, x^{0}\right)=\left\{\begin{array}{l}
v: \mathbb{R}^{n}: \forall\left\{x^{k}\right\} \longrightarrow x^{0}, \text { with } x^{k} \in X, \forall\left\{t_{k}\right\} \longrightarrow 0^{+}, \\
\exists\left\{v^{k}\right\} \longrightarrow v \text { with } x^{k}+t_{k} v^{k} \in X
\end{array}\right\},
$$


or equivalently,

$$
T^{C}\left(X, x^{0}\right)=\left\{\begin{array}{l}
v: \mathbb{R}^{n}: \forall N(v), \exists N\left(x^{0}\right), \exists \lambda>0 \text { such that } \forall t \in(0, \lambda), \\
\forall \bar{x} \in N\left(x^{0}\right) \cap X, \exists \bar{v} \in N(v) \text { with } \bar{x}+t \bar{v} \in X
\end{array}\right\} .
$$

It is well-known that this cone is closed and convex and that $T^{C}\left(X, x^{0}\right) \subseteq T\left(X, x^{0}\right)$. Moreover, if $f$ is continuously differentiable in a neighborhood of $x^{0}$, then $\partial^{C} f\left(x^{0}\right)=\left\{\nabla f\left(x^{0}\right)\right\}$. So, in this case we have another Fritz John-type optimality condition for $(P)_{3}$. Moreover, if $X$ is a closed convex set, then the Clarke normal cone collapses to the classical normal cone of the Convex Analysis, and therefore relation a) of Theorem 10 becomes relation a) of Theorem 5, again without the requirement $\operatorname{int}(X) \neq \varnothing$.

Besides the fruitful approach of Clarke $(1976,1983)$, problem $(P)_{3}$ has been considered also by Hestenes (1975), who makes use of the so-called "derived sets", which suitably approximate the image of $X$ under the mapping

$$
x \in X \longrightarrow\left(f(x), g_{1}(x), \ldots, g_{m}(x), h_{1}(x), \ldots, h_{r}(x)\right)
$$

near the image of the solution of $(P)_{3}$. We point out a paper of Breckner and Kolumban (1988), in which these authors overcome some difficulties and restrictions imposed by Hestenes.

\section{Vector Optimization with a Set Constraint. Basic Results}

We first introduce the usual order relations in $\mathbb{R}^{n}$. If $x \in \mathbb{R}^{n}$ and $y \in \mathbb{R}^{n}$, then:

- $x=y \Longleftrightarrow x_{i}=y_{i}, i=1, \ldots, n$;

- $x<y \Longleftrightarrow x_{i}<y_{i}, i=1, \ldots, n$;

- $x \leqq y \Longleftrightarrow x_{i} \leqq y_{i}, i=1, \ldots, n$;

- $x \leq y \Longleftrightarrow x \leqq y$, but $x \neq y$; i. e. $x \leqq y$ but there exists an index $i$ such that $x_{i}<y_{i}$.

In this Section we shall consider the following vector optimization problems or Pareto optimization problems or multiobjective optimization problems.

$$
\begin{aligned}
& (V P)_{1}: \quad V-\min f(x), x \in X \\
& (V P)_{2}: \quad\left\{\begin{array}{l}
V-\min f(x) \\
g_{i}(x) \leqq 0, i=1, \ldots, m, \\
x \in X
\end{array}\right. \\
& (V P)_{3}: \quad\left\{\begin{array}{l}
V-\min f(x) \\
g_{i}(x) \leqq 0, i=1, \ldots, m, \\
h_{j}(x)=0, j=1, \ldots, r<n, \\
x \in X,
\end{array}\right.
\end{aligned}
$$

where $X$ is a subset of $\mathbb{R}^{n}, f: \Omega \longrightarrow \mathbb{R}^{p}, g: \Omega \longrightarrow \mathbb{R}^{m}, h: \Omega \longrightarrow \mathbb{R}^{r}, \Omega$ open set of $\mathbb{R}^{n}$ containing $X, f$ and $g$ differentiable on $\Omega$ and $h$ continuously differentiable on $\Omega$.

Definition 1. A feasible point $x^{0}$ for $(V P)_{1}$ is said to be an efficient point or efficient solution or Pareto optimal point for $(V P)_{1}$ if there does not exist another point $x \in X$ such that $f(x) \leq f\left(x^{0}\right)$. In other words, if

$$
f(x)-f\left(x^{0}\right) \notin-\mathbb{R}_{+}^{p} \backslash\{0\}, \quad \forall x \in X .
$$

Definition 2. A feasible point $x^{0}$ for $(V P)_{1}$ is said to be a weakly efficient point or weakly efficient solution or weak Pareto optimal point for $(V P)_{1}$ if there does not exist another point $x \in X$ such that $f(x)<f\left(x^{0}\right)$. In other words, if

$$
f(x)-f\left(x^{0}\right) \notin-\operatorname{int}\left(\mathbb{R}_{+}^{p}\right), \quad \forall x \in X .
$$

If the previous conditions are verified in $U\left(x^{0}\right) \cap X$, where $U\left(x^{0}\right)$ is a suitable neighborhood of $x^{0}$, then $x^{0}$ is said to be a local efficient point or a local weakly efficient point, respectively. Obviously (local) efficiency implies (local) weak efficiency. In the scalar case ( $p=1)$ a weakly efficient (local weakly efficient) and an efficient point (local efficient point) collapse to the usual definition of a minimum (local minimum) point.

As we are concerned with necessary optimality conditions for $(V P)_{i}, i=1,2,3$, usually we shall make reference to the concept of weakly efficient points. The following necessary optimality conditions for $(V P)_{1}$ hold.

Theorem 11. If $x^{0} \in X$ is a local weakly efficient point for $(V P)_{1}$, then

$$
T\left(X, x^{0}\right) \cap F_{0}=\varnothing,
$$


where

$$
F_{0}=\left\{v \in \mathbb{R}^{n}: J f\left(x^{0}\right) v \in-i n t\left(\mathbb{R}_{+}^{p}\right)\right\}
$$

In other words, the system

$$
J f\left(x^{0}\right) v<0
$$

has no solutions $v \in T\left(X, x^{0}\right)$.

Proof. See Corley (1985), Jimenez and Novo (2004), Giorgi and Zuccotti (2012).

Remark 5. Another way to express Theorem 11 is the following one: if $x^{0}$ is a local weakly efficient point for $(V P)_{1}$, then

$$
\max _{i=1, \ldots, p}\left[\nabla f_{i}\left(x^{0}\right) v\right] \geqq 0
$$

for all $v \in T\left(X, x^{0}\right)$. In the single-objective case this condition holds at an optimal point considering any direction $v$ in the closed convex hull of $T\left(X, x^{0}\right)$, i. e. in the cone $P\left(X, x^{0}\right)$. This is no longer true in the multiobjective case, as Wang and Yang (1991) have shown. However (see Castellani and Pappalardo (2001)), if $f$ is convexlike on $X$, then if $x^{0} \in X$ is a weakly efficient point for $(V P)_{1}$, then

$$
\max _{i=1, \ldots, p}\left[\nabla f_{i}\left(x^{0}\right) v\right] \geqq 0, \quad \forall v \in P\left(X, x^{0}\right),
$$

or, equivalently, by the strict separation theorem,

$$
\operatorname{conv}\left\{\nabla f_{i}\left(x^{0}\right), i=1, \ldots, p\right\} \cap\left(T\left(X, x^{0}\right)\right)^{*} \neq \varnothing .
$$

Remark 6. If $x^{0} \in \operatorname{int}(X)$, then $T\left(X, x^{0}\right)=\mathbb{R}^{n}$ and the thesis of Theorem 11 becomes: the system

$$
J f\left(x^{0}\right) v<0
$$

has no solution $v \in \mathbb{R}^{n}$. Applying the Gordan theorem of the alternative (see, e. g., Mangasarian (1969)), this is equivalent to the proposition: there exists $y \in \mathbb{R}^{p}$ such that

$$
y J f\left(x^{0}\right)=0, y \geq 0 .
$$

Condition (14) was obtained, in an autonomous way, by Craven (1977); a feasible vector $x^{0}$ satisfying (14), is also called a "vector critical point" for $(V P)_{1}$.

We now consider $(V P)_{2}$, i. e. a vector optimization problem with only inequality constraints and an abstract constraint. For this case Jiménez and Novo (2002b) have proved the following results, which "parallel" the corresponding results of Theorem 4, established for scalar problems.

Theorem 12. Let $x^{0}$ be a local weakly efficient point for $(V P)_{2}$ and assume that $T\left(X, x^{0}\right)$ is a convex cone. Then, there exist multipliers $\alpha_{s}, s=1, \ldots, p, \lambda_{i}, i=1, \ldots, m$, not all zero, such that

$$
\begin{gathered}
0 \in \sum_{s=1}^{p} \alpha_{s} \nabla f_{s}\left(x^{0}\right)+\sum_{i=1}^{m} \nabla g_{i}\left(x^{0}\right)+\left(T\left(X, x^{0}\right)\right)^{*} \\
\alpha_{s} \geqq 0, s=1, \ldots, p ; \quad \lambda_{i} \geqq 0, i=1, \ldots, m ; \\
\lambda_{i} g_{i}\left(x^{0}\right)=0, \quad i=1, \ldots, m .
\end{gathered}
$$

Remark 7. A similar result holds with reference to any convex subcone $T_{1}\left(X, x^{0}\right)$ of $T\left(X, x^{0}\right)$, with vertex at $0 \in T_{1}\left(X, x^{0}\right)$. Under the assumptions of the previous theorem, if, moreover, it holds the following constraint qualification

$$
G_{0}^{+} \cap T\left(X, x^{0}\right) \neq \varnothing
$$

or

$$
G_{0}^{+} \cap T_{1}\left(X, x^{0}\right) \neq \varnothing
$$

where

$$
G_{0}^{+}=\left\{v \in \mathbb{R}^{n}: \nabla g_{i}\left(x^{0}\right) v<0, \quad i \in I\left(x^{0}\right)\right\},
$$

then (15) holds with $\alpha=\left[\alpha_{1}, \ldots, \alpha_{s}\right] \neq 0$, i. e. with $\alpha \geq 0$. 
If $X$ is a convex set, then $T\left(X, x^{0}\right)$ is convex and in relation (15) the cone $\left(T\left(X, x^{0}\right)\right)^{*}$ equals the normal cone $N\left(X, x^{0}\right)$. Moreover, in this case relation (16) can be substituted with the equivalent condition

$$
G_{0}^{+} \cap\left(X-x^{0}\right) \neq \varnothing .
$$

If we assume that $f$ and $g$ are convex, $X$ is a convex set, $x^{0}$ is an efficient solution for $(V P)_{2}$ and that the weak Slater constraint qualification holds:

$$
\text { there exists } \bar{x} \in X \text { such that } g_{i}(\bar{x})<0, i \in I\left(x^{0}\right) \text {, }
$$

then (15) holds with $\alpha \neq \varnothing$ (i. e. with $\alpha \geq 0$ ). If, moreover, we assume that the strong Slater constraint qualification holds:

$$
\begin{gathered}
\text { for each } s=1, \ldots, p \text {, there exist } \bar{x}_{s} \text { such that } \\
f_{k}\left(\bar{x}_{s}\right)<f_{k}\left(x^{0}\right), \forall k \neq s, g_{i}\left(\bar{x}_{s}\right)<0, \forall i \in I\left(x^{0}\right), \bar{x}_{s} \in X,
\end{gathered}
$$

then (15) holds with $\alpha>0$.

We now consider $(V P)_{3}$; this vector optimization problem has been treated by several authors, but here we follow mainly the approach of Giorgi, Jiménez and Novo (2004), Jiménez and Novo (2002a) and Jiménez and Novo (2002b). We first treat the case of a convex constraint set $X$.

A) The constraint set $X$ is a convex set.

We do not assume (contrary, e. g. to Jahn (2011)) that $\operatorname{int}(X) \neq \varnothing$. Also Robinson (1976, Theorem 3) obtains a minimum principle-type optimality condition for $(V P)_{3}$, with a convex constraint set $X$ and in infinite spaces for Fréchet differentiable functions (and without the condition $\operatorname{int}(X) \neq \varnothing$ ), by means of a "separation property" (Robinson (1976), Definition 5). In finite-dimensional spaces it is possible to obtain the following results (see the above quoted papers of Jiménez and Novo).

Theorem 13. Let $X \subseteq \mathbb{R}^{n}$ be a convex set and let $x^{0}$ be a local weakly efficient point for $(V P)_{3}$. Then, there exist multipliers $\alpha_{s}, s=1, \ldots, p ; \lambda_{i}, i=1, \ldots, m ; \mu_{j}, j=1, \ldots, r$, not all zero, such that

i) $\quad 0 \in \sum_{s=1}^{p} \alpha_{s} \nabla f_{s}\left(x^{0}\right)+\sum_{i=1}^{m} \lambda_{i} \nabla g_{i}\left(x^{0}\right)+\sum_{j=1}^{r} \mu_{j} \nabla h_{j}\left(x^{0}\right)+N\left(X, x^{0}\right)$;

ii) $\quad \lambda_{i} g_{i}\left(x^{0}\right)=0, i=1, \ldots, m$;

iii) $\quad(\alpha, \lambda) \geqq 0$.

Remark 8. If we impose some "regularity condition", we can obtain that iii) of the previous theorem holds in the form $(\alpha, \lambda) \geq 0$. This is assured, for example, by the condition:

$$
0 \in \sum_{j=1}^{r} \mu_{j} \nabla h_{j}\left(x^{0}\right)+N\left(X, x^{0}\right) \Longrightarrow \mu=0 .
$$

If, in addition to (17), we impose also the following constraint qualification:

$$
G_{0}^{+} \cap H_{0} \cap\left(X-x^{0}\right) \neq \varnothing,
$$

then $i$ ) of the previous theorem holds with $\alpha \geq 0$, i. e. we obtain for $(V P)_{3}$ the so-called Karush-Kuhn-Tucker conditions (in a weak form). Other constraint qualifications assuring $\alpha \geq 0$ in $i$ ) of Theorem 13, are presented in Giorgi, Jiménez and Novo (2004).

B) The constraint set $X$ is an arbitrary set of $\mathbb{R}^{n}$.

As for the scalar case, in order to obtain a minimum principle-type optimality condition for $(V P)_{3}$, we have to make use of the cone of interior directions $I\left(X, x^{0}\right)$ or better, of the cone of quasi-interior directions $Q\left(X, x^{0}\right)$.

Theorem 14. Let $X \subseteq \mathbb{R}^{n}$ be an arbitrary set and let $x^{0}$ be a local weakly efficient point for $(V P)_{3}$. Let $Q\left(X, x^{0}\right)$ be a convex cone; then there exist multipliers $\alpha_{s}, s=1, \ldots, p ; \lambda_{i}, i=1, \ldots, m ; \mu_{j}, j=1, \ldots, r$, not all zero, such that

a) $0 \in \sum_{s=1}^{p} \alpha_{s} \nabla f_{s}\left(x^{0}\right)+\sum_{i=1}^{m} \lambda_{i} \nabla g_{i}\left(x^{0}\right)+\sum_{j=1}^{r} \mu_{j} \nabla h_{j}\left(x^{0}\right)+\left(Q\left(X, x^{0}\right)\right)^{*}$;

b) $\lambda_{i} g_{i}\left(x^{0}\right)=0, i=1, \ldots, m$;

c) $(\alpha, \lambda) \geqq 0$.

Remark 9. Under the assumptions of Theorem 14, if, moreover,

$$
G_{0}^{+} \cap H_{0} \cap Q\left(X, x^{0}\right) \neq \varnothing
$$


and the Jacobian matrix $J h\left(x^{0}\right)$ has full rank, then a) of the previous theorem holds with $\alpha \geq 0$.

Remark 10. It is possible to obtain for $(V P)_{3}$ minimum principle-type optimality conditions with the use of the Bouligand tangent cone $T\left(X, x^{0}\right)$, instead of the cone $I\left(X, x^{0}\right)$ or the cone $Q\left(X, x^{0}\right)$, by imposing a suitable regularity condition or constraint qualification, as shown in Jiménez and Novo (2002b) and in Giorgi, Jiménez and Novo (2004).

Theorem 15. Let $x^{0}$ be a local weakly efficient solution for $(V P)_{3}$ and let

a) either $T\left(X, x^{0}\right)$ be convex,

b) or $T_{1}\left(X, x^{0}\right)$ be convex, where $T_{1}\left(X, x^{0}\right)$ is any subcone of $T\left(X, x^{0}\right)$, with $0 \in T_{1}\left(X, x^{0}\right)$.

Assume the following "extended Abadie constraint qualification":

$$
G_{0} \cap H_{0} \cap T\left(X, x^{0}\right) \subseteq T\left(C \cap X, x^{0}\right),
$$

where $C=A \cap B=\left\{x \in \mathbb{R}^{n}: g_{i}(x) \leqq 0, i=1, \ldots, m\right\} \cap\left\{x \in \mathbb{R}^{n}: h_{j}(x)=0, j=1, \ldots, r\right\}$.

(If we use the convex subcone $T_{1}\left(X, x^{0}\right)$, in the previous relation the cone $T\left(X, x^{0}\right)$ has to be substituted with $T_{1}\left(X, x^{0}\right)$ ).

Then, there exist multipliers $\alpha_{s}, s=1, \ldots, p ; \lambda_{i}, i=1, \ldots, m ; \mu_{j}, j=1, \ldots, r$, not all zero, such that

i) $\quad 0 \in \sum_{s=1}^{p} \alpha_{s} \nabla f_{s}\left(x^{0}\right)+\sum_{i=1}^{m} \lambda_{i} \nabla g_{i}\left(x^{0}\right)+\sum_{j=1}^{r} \mu_{j} \nabla h_{j}\left(x^{0}\right)+\left(T\left(X, x^{0}\right)\right)^{*}$;

ii) $\quad \lambda_{i} g_{i}\left(x^{0}\right)=0, i=1, \ldots, m$;

iii) $\quad(\alpha, \lambda) \geqq 0$.

Remark 11. If $\operatorname{lin}\left\{\nabla h_{j}\left(x^{0}\right), j=1, \ldots, r\right\}+\left(T\left(X, x^{0}\right)\right)^{*}$ is closed, then $\left.i\right)$ is satisfied with $(\alpha, \lambda) \neq 0(\operatorname{lin}\{S\}$ is the linear span of $S$ ).

If cone conv $\left\{\nabla g_{i}\left(x^{0}\right), i \in I\left(x^{0}\right)\right\}+\operatorname{lin}\left\{\nabla h_{j}\left(x^{0}\right), j=1, \ldots, r\right\}+\left(T\left(X, x^{0}\right)\right)^{*}$ is closed, then $\left.i\right)$ is satisfied with $\alpha \neq 0$. A sufficient condition for the validity of the last "closedness" relation is:

$$
G_{0}^{+} \cap H_{0} \cap \operatorname{relint}\left(T\left(X, x^{0}\right)\right) \neq \varnothing .
$$

Bender (1978) uses, instead of the "extended Abadie constraint qualification", another regularity condition, i. e.

$$
H_{0} \cap T\left(X, x^{0}\right) \subseteq T\left(B \cap X, x^{0}\right) .
$$

Obviously, if the inequality constraints are not considered, the extended Abadie constraint qualification collapses to the Bender constraint qualification. However, if the inequality constraint are present, the two conditions are not comparable. See Jimenéz and Novo (2002b).

Remark 12. Giorgi, Jiménez and Novo (2004) consider $(V P)_{3}$, with $X$ an arbitrary set constraint, and by means of an "extended Bender constraint qualification" they obtain the following minimum principle-type optimality conditions (under weaker differentiability assumptions than the ones of the present paper).

Theorem 16. Let in $(V P)_{3}$ be $X$ an arbitrary set of $\mathbb{R}^{n}$ and let $P \subseteq \mathbb{R}^{n}$ be a convex set with $0 \in P$. Let the following "extended Bender constraint qualification" hold:

$$
H_{0} \cap P \subseteq T\left(B \cap X, x^{0}\right) .
$$

If $x^{0}$ is a local weakly efficient solution of $(V P)_{3}$, then there exist multipliers $\alpha_{s}, s=1, \ldots, p ; \lambda_{i}, i=1, \ldots, m ; \mu_{j}, j=1, \ldots, r$, not all zero, such that

1) $0 \in \sum_{s=1}^{p} \alpha_{s} \nabla f_{s}\left(x^{0}\right)+\sum_{i=1}^{m} \lambda_{i} \nabla g_{i}\left(x^{0}\right)+\sum_{j=1}^{r} \mu_{j} \nabla h_{j}\left(x^{0}\right)+P^{*}$;

2) $\lambda_{i} g_{i}\left(x^{0}\right)=0, i=1, \ldots, m$;

3) $(\alpha, \lambda) \geqq 0$.

Remark 13. We obtain the same conclusions of Theorem 16, by choosing $P=I\left(X, x^{0}\right) \cup\{0\}$ or $P=Q\left(X, x^{0}\right) \cup\{0\}$, with $I\left(X, x^{0}\right)$ and $Q\left(X, x^{0}\right)$ convex, without assuming the extended Bender constraint qualification (see Giorgi, Jiménez and Novo (2004)). However, we note once again that if $\operatorname{int}(X)=\varnothing$, we have $I\left(X, x^{0}\right)=Q\left(X, x^{0}\right)=\varnothing$, and so $\left(I\left(X, x^{0}\right)\right)^{*}=$ $\left(Q\left(X, x^{0}\right)\right)^{*}=\mathbb{R}^{n}$, therefore condition 1$)$ of Theorem 16 becomes trivially satisfied and not useful.

On the other hand, the Bender constraint qualification allows to deduce directly form Theorem 16 the minimum principletype optimality conditions for the case of $X$ convex, without assuming $\operatorname{int}(X) \neq \varnothing$. Indeed. if $X$ is convex and regularity 
condition (17) holds, i. e.

$$
\text { (RC) } \quad 0 \in \sum_{j=1}^{r} \mu_{j} \nabla h_{j}\left(x^{0}\right)+N\left(X, x^{0}\right) \Longrightarrow \mu=0,
$$

Jiménez and Novo (2002a) have proved that we have

$$
H_{0} \cap T\left(X, x^{0}\right)=T\left(B \cap X, x^{0}\right),
$$

i. e. a Bender constraint qualification (where $P=T\left(X, x^{0}\right)$, convex cone, being $X$ convex). If (RC) does not hold, it is always possible to choose multipliers $\mu_{j}$, not all zero, such that the thesis of Theorem 16 holds. A similar approach is also used by R. Cambini: see the next Section.

\section{Vector Optimization with a Set Constraint. The Approach of R. Cambini}

Riccardo Cambini has been concerned with vector optimization problems with a set constraint in several papers; see Cambini $(1998,1999,2002,2003,2007)$. This author studies a vector optimization problem more general than $(V P)_{3}$, in the sense that the ordering cone is not necessarily the Paretian cone, but it is given by a closed convex pointed cone with a nonempty interior. Moreover, the differentiability assumptions on the functions involved in the said problem are more general than ours, as also the Hadamard directional differentiability is taken into account and, finally, the optimality conditions are expressed both in the image space and in the decision space. We shall give a brief account of Cambini's results, referred to $(V P)_{3}$ under Fréchet differentiability and continuous Fréchet differentiability, following the usual decision space approach.

Theorem 17. Consider problem $(V P)_{3}$ and assume $f$ and $g$ to be Fréchet differentiable at the feasible point $x^{0}$. Moreover, let $h$ be continuously differentiable in a neighborhood of $x^{0}$. Let $U \subseteq \mathbb{R}^{n}$ be a cone which verifies one of the following regularity conditions:

R1) $\quad U$ is convex and

$$
U \cap T\left(B, x^{0}\right) \subseteq T\left(B \cap X, x^{0}\right) ;
$$

R2) $\quad U=I\left(X, x^{0}\right)$ is convex;

R3) $U=T\left(X, x^{0}\right)$ and $X$ is convex.

If the feasible point $x^{0}$ is a local efficient point for $(V P)_{3}$, then there exist multipliers $\alpha_{s} \geqq 0, s=1, \ldots, p ; \lambda_{i} \geqq 0$, $i=1, \ldots, m ; \mu_{j} \in \mathbb{R}$, not all zero, such that

$$
\begin{gathered}
\lambda_{i} g_{i}\left(x^{0}\right)=0, \quad i=1, \ldots, m \\
\left(\sum_{s=1}^{p} \alpha_{s} \nabla f_{s}\left(x^{0}\right)+\sum_{i=1}^{m} \lambda_{i} \nabla g_{i}\left(x^{0}\right)+\sum_{j=1}^{r} \mu_{j} \nabla h_{j}\left(x^{0}\right)\right) v \geqq 0, \quad \forall v \in \operatorname{cl}(U) .
\end{gathered}
$$

Proof. See Cambini (2007).

Remark 14. The previous results hold also if $x^{0}$ is a local weakly efficient point for $(V P)_{3}$. If $X$ is convex and the feasible point $x^{0}$ is a local efficient point for $(V P)_{3}$, then the following minimum principle-type optimality conditions hold:

There exist multipliers $\alpha_{s} \geqq 0, s=1, \ldots, p ; \lambda_{i} \geqq 0, i=1, \ldots, m ; \mu_{j} \in \mathbb{R}$, not all zero, such that $\lambda_{i} g_{i}\left(x^{0}\right)=0, i=1, \ldots, m$;

$$
\left(\sum_{s=1}^{p} \alpha_{s} \nabla f_{s}\left(x^{0}\right)+\sum_{i=1}^{m} \lambda_{i} \nabla g_{i}\left(x^{0}\right)+\sum_{j=1}^{r} \mu_{j} \nabla h_{j}\left(x^{0}\right)\right)\left(y-x^{0}\right) \geqq 0, \quad \forall y \in X .
$$

Indeed, this result follows at once from $R 3$ ) of Theorem 17, by choosing $U=F\left(X, x^{0}\right) \subseteq T\left(X, x^{0}\right)$. Note that the assumption $\operatorname{int}(X) \neq \varnothing$ is not required. Obviously, if $x^{0} \in \operatorname{int}(X)$ all the minimum principle-type necessary optimality conditions taken into consideration in the present paper collapse to the usual Fritz John conditions or to the usual Karush-KuhnTucker conditions.

\section{References}

Aubin, J. P., \& Frankowska, H. (1990). Set-Valued Analysis, Birkhäuser, Boston.

Bazaraa, M. S., \& Goode, J. J. (1972). Necessary optimality criteria in mathematical programming in the presence of differentiability, J. Math. Anal. Appl., 40, 609-621. https://doi.org/10.1016/0022-247X(72)90006-6

Bazaraa, M. S., Goode, J. J., \& Nashed, M. S. (1974). On the cone of tangents with applications to mathematical programming, J. Optim. Theory Appl., 13, 389-426. https://doi.org/10.1007/BF00934938 
Bazaraa, M. S., \& Shetty, C. M. (1976). Foundations of Optimization, Springer Verlag, New York. https://doi.org/10.1007/978-3-642-48294-6

Bender, P. J. (1978). Nonlinear programming in normed linear spaces, J. Optim. Theory Appl., 24, $263-285$. https://doi.org/10.1007/BF00933281

Bertsekas, D. P., \& Ozdaglar, A. E. (2002). Pseudonormality and a Lagrange multiplier theory for constrained optimization, J. Optim. Theory Appl., 114, 287-343.

Breckner, W. W., \& Kolumban, I. (1988). Multiplier rules for optimization problems with a finite number of constraints, Studia Univ. Babes-Bolyai, Mathematica, 33, 15-37.

Cambini, R. (1998). Minimum principle type necessary optimality conditions: preliminary results; in Giorgi, G. \& Rossi, F. (Eds.), Convessità e Calcolo Parallelo, Libreria Universitaria Editrice, Verona, 65-79.

Cambini, R. (1999). Minimum principle type optimality conditions with equality constraints: preliminary results; in Giorgi, G. \& Rossi, F. (Eds.), Generalized Convexity for Economics and Financial Decisions, Pitagora Editrice, Bologna, 63-79.

Cambini, R. (2002). Developments on $U$-regularity conditions; in Crespi, G. \& others (Eds.), Optimization in Economics, Finance and Industry, Datanova Editrice, Milan, 103-117.

Cambini, R. (2003). Multiobjective problems with set constraints; in Crespi, G. \& others (Eds.), Recent Advances in Optimization, Datanova Editrice, Milan, 1-20.

Cambini, R. (2007). Multiobjective problems with set constraints: from necessary optimality conditions to duality results; in Allevi, A. \& others (Eds.), Nonlinear Analysis with Applications in Economics, Energy and Transportation, Bergamo Univ. Press, Bergamo, 25-64.

Canon, M., Cullum, C., \& Polak, E. (1966). Constrained minimization problems in finite-dimensional spaces, SIAM J. on Control, 4, 528-547.

Canon, M., Cullum, C., \& Polak, E. (1970). Theory of Optimal Control and Mathematical Programming, McGraw-Hill Book Company, New York.

Castellani, M., \& Pappalardo, M. (2001). About a gap between multiobjective optimization and scalar optimization, J. Optim. Theory Appl., 109, 437-439. https://doi.org/10.1023/A:1017526724872

Clarke, F. H. (1976). A new approach to Lagrange multipliers, Mathematics of Operations Research, 1, 165-174. https://doi.org/10.1287/moor.1.2.165

Clarke, F. H. (1983). Optimization and Nonsmooth Analysis, J. Wiley, New York.

Corley, H. W. (1985). On optimality conditions for maximization with respect to cones, J. Optim. Theory Appl., 46, 67-78. https://doi.org/10.1007/BF00938760

Craven, B. D. (1977). Lagrangian conditions and quasiduality, Bulletin of the Australian Mathematical Society, 16, $325-$ 339. https://doi.org/10.1017/S0004972700023431

Di, S. (1996). Classical optimality conditions under weaker assumptions, SIAM J. Optimization, 6, 178-197.

Di, S., \& Poliquin, R. (1994). Contingent cone to a set defined by equality and inequality constraints at a Fréchet differentiable point, J. Optim. Theory Appl., 81, 469-478. https://doi.org/10.1007/BF02193096

Flores-Bazan, F. (2014). Fritz John necessary optimality conditions of the alternative-type, J. Optim. Theory Appl., 161, 807-818.

Flores-Bazan, F., \& Mastroeni, G. (2015). Characterizing FJ and KKT conditions in nonconvex mathematical programming with applications, SIAM J. Optimization, 25, 647-676. https://doi.org/10.1137/13094606X

Giorgi, G., \& Guerraggio, A. (1992). On the notion of tangent cone in mathematical programming, Optimization, 25, 11-23. https://doi.org/10.1080/02331939208843804

Giorgi, G., \& Guerraggio, A. (1994). First order generalized optimality conditions for programming problems with a set constraint; in Komlosi, S., Rapcsàk, T. \& Schaible, S. (Eds.), Generalized Convexity. Proceedings of the IVth International Workshop on Generalized Convexity Held at Janus Pannonius University, Pècs, Hungary, August 31-September 2, 1992, Springer, Berlin, 171-185. https://doi.org/10.1007/978-3-642-46802-5-15

Giorgi, G., \& Guerraggio, A. (2002). Characterizations, comparisons, algebraic and topological properties of tangent 
cones, Journal of Statistics $\mathcal{E}$ Management Systems, 5, 275-294.

Giorgi, G., Jiménez, B. \& Novo, V. (2004). Minimum principle-type optimality conditions for Pareto problems, Int. Journal of Pure and Applied Mathematics, 10, 51-68.

Giorgi, G., \& Zuccotti, C. (2012). On the use of tangent cones and sets in vector optimization, Dipartimento di Economia Politica e Metodi Quantitativi, Università di Pavia, Report N. 169 (04-12).

Gould, F. J., \& Tolle, J. W. (1971). A necessary and sufficient qualification for constrained optimization, SIAM J. Appl. Math., 20, 164-172. https://doi.org/10.1137/0120021

Gould, F. J., \& Tolle, J. W. (1972). Geometry of optimality conditions and constraint qualifications, Math. Programming, 2, $1-18$

Guignard, M. (1969). Generalized Kuhn-Tucker conditions for mathematical programming problems in a Banach space, SIAM J. on Control, 7, 232-241.

Halkin, H. (1966). A maximum principle of the Pontryagin type for systems described by nonlinear difference equations, SIAM J. on Control, 4. 90-111. https://doi.org/10.1073/pnas.56.4.1066

Halkin, H., \& Neustadt, L. W. (1966). General necessary conditions for optimization problems, Proc. Nat. Acad. Sci. U. S., 56, 1066-1071.

Hestenes, M. R. (1966). Calculus of Variations and Optimal Control Theory, John Wiley \& Sons, New York.

Hestenes, M. R. (1975). Optimization Theory. The Finite Dimensional Case, John Wiley \& Sons, New York.

Jahn, J. (1996). Introduction to the Theory of Nonlinear Optimization - Second Revised Edition, Springer, New York. https://doi.org/10.1007/978-3-662-03271-8

Jahn, J. (2011). Vector Optimization: Theory, Applications, Extensions - Second Edition, Springer, New York.

Jiménez, B., \& Novo, V. (2002a). A finite dimensional extension of Lyusternik theorem with applications to multiobjective optimization, J. Math. Anal. Appl., 270, 340-356.

Jiménez, B. \& Novo, V. (2002b). Alternative theorems and necessary optimality conditions for directionally differentiable multiobjective programs, J. of Convex Analysis, 9, 97-116.

Jiménez, B., \& Novo, V. (2004). Optimality conditions in differentiable vector optimization via second-order tangent sets, Appl. Math. Optim., 49, 123-144.

Kirsch. A., Warth, W., \& Werner, J. (1978). Notwendige Optimalitatsbedingungen und ihre Anwendung, Springer, Berlin.

Mangasarian, O. L. (1969). Nonlinear Programming, McGraw-Hill, New York.

Mangasarian, O. L., \& Fromovitz S. (1967). The Fritz John necessary optimality conditions in the presence of eqaulity and inequality constraints, J. Math. Anal. Appl., 17, 37-47.

Martin. D. H., Gardner, R. J., \& Watkins, G. G. (1981). Indicating cones and the intersection principle for tangential approximants in abstract multiplier rules, J. Optim. Theory Appl., 33, 515-537.

Martin, D. H., \& Watkins, G. G. (1985). Cores of tangent cones and Clarke's tangent cone, Mathematics of Oper. Res., $10,565-575$.

Michel, P., \& Penot, J. P. (1984). Calcul sous-différentiel pour des fonctions Lipshitziennes et non-Lipshitziennes, $C$. $R$. Acad. Sciences, Paris, Série I, Mathématique, 298, 269-272.

Mishra, L. N. (2017). On existence and behavior of solutions to some nonlinear integral equations with applications, Ph. D. Thesis, National Institute of Technology, Silchar 788010, Assam, India.

Mishra, V. N. (2007). Some problems on approximations of functions in Banach spaces, Ph. D. Thesis, Indian Institute of Technology, Roorkee 247667, Uttarakhand, India.

Mishra, V. N., \& Mishra, L. N. (2012). Trigonometric approximation of signals (functions) in $L_{p}$-norm, Int. J. Contemp. Math. Sciences, 7, 909-918.

Mordukhovich, B. (1976). Maximum principle in the problem of time optimal response with nonsmooth constraints, Journal of Applied Mathematics and Mechanics, 40, 960-969.

Mordukhovich, B. (2005). Variational Analysis anf Generalized Differentiation (2 Volumes). Springer, Berlin.

Nagahisa, Y. (1988). Necessary conditions for a mathematical programming problem with set and cone constraints, $J$. 
Austral. Math. Soc., Ser. B, 29, 310-321.

Nagahisa, Y., \& Sakawa, Y. (1969). Nonlinear programming in Banach spaces, J. Optim. Theory Appl., 4, $182-190$.

Neustadt, L. (1969). A general theory of extremals, J. of Computer and System Science, 3, 57-92. https://doi.org/10.1016/S0022-0000(69)80007-3

Neustadt, L. (1976). Optimization: A Theory of Necessary Conditions, Princeton Univ. Press, Princeton.

Pathak, H. K., \& Deepmala (2012). Some existence theorems for solvability of certain functional equations arising in dynamic programming, Bull. Cal. Math. Soc., 104 (3), 237-244.

Pathak, H. K., \& Deepmala (2012). Existence and uniqueness of solutions of functional equations arising in dynamic programming, Applied Mathematics and Computation, 218 (13), 7721-7230.

Ponstein, J. (1980). Approaches to the Theory of Optimization, Cambridge Univ. Press, Cambridge.

Pshenichnyi, B. N. (1971). Necessary Conditions for an Extremum, M. Dekker, New York.

Pshenichnyi, B. N., \& Danilin, Yu. (1978). Numerical Methods in Extremal Problems, MIR Publishers, Moscow.

Robinson, S. (1976). First order conditions for general nonlinear optimization, SIAM J. Appl. Math., 30, $597-607$.

Rockafellar, R. T. (1993). Lagrange multipliers and optimality, SIAM Review, 35, 183-238.

Rockafellar, R. T., \& Wets, R., J.-B. (1998). Variational Analysis, Springer, Berlin (corrected $3^{\text {rd }}$ printing: 2009).

Treiman, J. S. (1991). An infinite class of convex tangent cones, J. Optim. Theory Appl., 68, 563-581. https://doi.org/10.1007/BF00940070

Varaiya, P. P. (1967). Nonlinear programming in Banach space, SIAM J. Appl. Math., 15, 284-293. https://doi.org/10.1137/0115028

Wang, S. Y., \& Yang, F. M. (1991). A gap between multiobjective optimization and scalar optimization, J. Optim. Theory Appl., 68, 389-391. https://doi.org/10.1007/BF00941577

Ward, D. (1987). Convex subcones of the contingent cone in nonsmooth calculus and optimization, Trans. Amer. Math. Soc., 302, 661-682. (Corrigendum in Trans. Amer. Math. Soc., 311, 429-431, 1989). https://doi.org/10.1090/S00029947-1987-0891640-2

Werner, J. (1984). Optimization Theory and Applications, F. Vieweg \& Sohn, Braunsheig/ Wiesbaden.

\section{Copyrights}

Copyright for this article is retained by the author(s), with first publication rights granted to the journal.

This is an open-access article distributed under the terms and conditions of the Creative Commons Attribution license (http://creativecommons.org/licenses/by/4.0/). 